\title{
The evolution of the spectrum of a Frobenius Lie algebra under deformation
}

\author{
Vincent E. Coll, Jr. ${ }^{*}$, Nicholas Mayers ${ }^{* *}$, and Nicholas Russoniello* \\ *Department of Mathematics, Lehigh University, Bethlehem, PA, 18015 \\ ** Department of Mathematics, Milwaukee School of Engineering, Milwaukee, WI, 53202
}

September 1, 2020

\begin{abstract}
The category of Frobenius Lie algebras is stable under deformation, and here we examine explicit infinitesimal deformations of four and six dimensional Frobenius Lie algebras with the goal of understanding if the spectrum of a Frobenius Lie algebra can evolve under deformation. It can.
\end{abstract}

Mathematics Subject Classification 2010: 17B20, 05E15

Key Words and Phrases: Frobenius Lie algebra, deformation, spectrum

\section{Introduction}

A Lie algebra $(\mathfrak{g},[-,-])$ is Frobenius if there exists a linear functional $F \in \mathfrak{g}^{*}$ such that the natural map $\eta: \mathfrak{g} \rightarrow \mathfrak{g}^{*}$ defined by $x \mapsto F[x,-]$ is an isomorphism. Such an $F$ is called a Frobenius functional. The set of Frobenius functionals of a Frobenius Lie algebra $\mathfrak{g}$ is, in general, quite large; forming an open subset of $\mathfrak{g}^{*}$ in the Zariski and Euclidean topologies (see [27] and [13]).

Frobenius Lie algebras were introduced in the 1970's by Ooms who showed, in particular, that the universal enveloping algebra $U(\mathfrak{g})$ admits a faithful simple representation when $\mathfrak{g}$ is Frobenius (see [27]). Such algebras also have applications in invariant theory and the geometry of coadjoint orbits in $\mathfrak{g}^{*}$ (see [25]). Deformation theorists are interested in Frobenius Lie algebras because each provides a solution to the classical Yang-Baxter equation, which in turn quantizes to a universal deformation formula, i.e., a Drinfel'd twist which deforms any algebra which admits an action of $\mathfrak{g}$ by derivations (see [21]).

If $\mathfrak{g}$ is Frobenius and $F \in \mathfrak{g}^{*}$ is a Frobenius functional, then the inverse image of $F$ under the mapping $\eta$ is called a principal element of $\mathfrak{g}$ and will be denoted $\widehat{F}$ (see [20]). It is the unique element of $\mathfrak{g}$ such that

$$
F \circ \text { ad } \widehat{F}=F([\widehat{F},-])=F \text {. }
$$

In [27], Ooms established that the spectrum of the adjoint of a principal element of a Frobenius Lie algebra is independent of the principal element chosen to compute it (see also [19]). Consequently, we can unambiguously refer to the spectrum of $\mathfrak{g}$ as the spectrum of the adjoint representation of any Frobenius functional $F \in \mathfrak{g}^{*}$.

Since the category of Frobenius Lie algebras is stable under deformation - the index can only decrease under deformation (see [1]) - we come to the motivating question of this article.

Q: Does the spectrum of a Frobenius Lie algebra evolve under the deformation of the underlying algebra?

To address this answer one requires easy examples of infinitesimal deformations of Frobenius Lie algebras. Such examples are sparse (see [22]). 
Here, following Czikoss and Verhoczkis' classification of four and six-dimensional Frobenius Lie algebras ([12], cf. Tables 1 and 2), we investigate the infinitesimal deformation theory of these algebras to find that many of these Frobenius Lie algebras can be deformed. A particularly rich example is provided by a certain one of these four-dimensional algebras, $\Phi^{\prime}$, for which we provide detailed cohomological and spectral calculations. The calculations are routine but potentially instructive.

The structure of the paper is as follows. In Section 2, we recall the well-known infinitesimal deformation theory of Nijenhuis and Richardson (see $[\mathbf{1 6}, \mathbf{1 7}]$ ), wherein the deformation of a Lie algebra is controlled by the graded Chevalley-Eilenberg complex (see [29]). In Section 3, we present the classification of fourdimensional Frobenius Lie algebras by detailing in Table 1 the commutator relations, the dimension of the second and third cohomology groups of the Lie algebra with coefficients in the Lie algebra (the case of interest for deformation theory), the spectrum, and whether or not deformations of the algebra exist. In Section 4, we provide detailed deformation theory calculations associated with $\Phi^{\prime}$ yielding Table 2 where we provide the full deformation and spectral values at deformation parameter instance $t$, for all deformations of the four-dimensional Lie algebras here considered. In Section 5, a short Epilogue provides connections between this paper's examples and topical "spectral" research, along with announcements of new results that will be appearing and will be of interest to researchers in this area. In Appendix A, Table 3 is the analogue of Table 2 for the six-dimensional Frobenius Lie algebras in Czikos's and Verhoczki's classification. The calculations are similar to those in the four-dimensional case but more tedious for the parametrized families. We provide the results of our cohomological calculations for one such parametrized family $\Phi_{6,12}(\xi)$ (see Example 1 ). We find this example interesting because for all values of $\xi$, $\operatorname{dim} H^{2}\left(\Phi_{6,12}(\xi) \neq 0\right.$ and only eight values of $\xi$ where $\operatorname{dim} H^{3}\left(\Phi_{6,12}(\xi), \Phi_{6,12}(\xi)\right) \neq 0$. Even so, all infinitesimals are unobstructed.

For the non-parametrized six-dimensional families, we provide the number of inequivalent deformations. As with the four-dimensional Lie algebras, all deformations are linear.

\section{Deformation theory}

Let $(\mathfrak{g},[]$,$) be a Lie algebra over an algebraically closed field \mathbb{F}$, where char $\mathbb{F} \neq 0$. A formal one-parameter deformation of $\mathfrak{g}$ is a power series

$$
[g, h]_{t}=[g, h]+\sum_{k \geq 1} \alpha_{k}(g, h) t^{k}
$$

where $\alpha_{k} \in \operatorname{HOM}_{\mathbb{F}}\left(\Lambda^{2} \mathfrak{g}, \mathfrak{g}\right)=\mathrm{C}^{2}(\mathfrak{g}, \mathfrak{g})$. The latter refers to the standard Chevalley-Eilenberg cochain complex $\left(C^{\bullet}(\mathfrak{g}, \mathfrak{g}), \delta\right)$ of $\mathfrak{g}$ with coefficients in the adjoint representation of $\mathfrak{g}$. Here, $C^{n}(\mathfrak{g}, \mathfrak{g})$ consists of forms $F^{n}$ : $\bigwedge_{i=1}^{n} \mathfrak{g} \rightarrow \mathfrak{g}$ satisfying $\delta^{2} F^{n}=0$, where the coboundary operator $\delta$ is defined by

$$
\begin{aligned}
\delta F^{n}\left(g_{1}, \ldots, g_{n+1}\right)=\sum_{i=1}^{n+1}(-1)^{i+1}[ & \left.g_{i}, F^{n}\left(g_{1}, \ldots, \hat{g}_{i}, \ldots, g_{n+1}\right)\right] \\
& +\sum_{1 \leq i<j \leq n+1}(-1)^{i+j} F^{n}\left(\left[x_{i}, x_{j}\right], x_{1}, \ldots, \hat{x}_{i}, \ldots, \hat{x}_{j}, \ldots, x_{n+1}\right) .
\end{aligned}
$$

In this setting, $Z^{n}(\mathfrak{g}, \mathfrak{g})=\operatorname{ker}(\delta) \cap C^{n}(\mathfrak{g}, \mathfrak{g}), B^{n}(\mathfrak{g}, \mathfrak{g})=\operatorname{Im}(\delta) \cap C^{n}(\mathfrak{g}, \mathfrak{g})$, and $H^{n}(\mathfrak{g}, \mathfrak{g})=Z^{n}(\mathfrak{g}, \mathfrak{g}) / B^{n}(\mathfrak{g}, \mathfrak{g})$. These comprise, respectively, the $n$-cocycles, $n$-coboundaries, and $n^{\text {th }}$ cohomology group of $\mathfrak{g}$ with coefficients in $\mathfrak{g}$. Of course, one requires that the deformation remains in the category of Lie algebras so that the Jacobi identity for $[,]_{t}$ is satisfied for all values of $t$. This is equivalent to the sequence of relations

$$
\delta \alpha_{k}=-\frac{1}{2} \sum_{i=1}^{k-1}\left[\alpha_{i}, \alpha_{k-i}\right]
$$


where

$$
\begin{aligned}
& {[\beta, \gamma]\left(g_{1}, \ldots, g_{p+q-1}\right)=\sum_{1 \leq i_{1}<\ldots<i_{q} \leq p+q-1}(-1)^{i_{1}+\ldots+i_{q}-\frac{q(q+1)}{2}} \beta\left(\gamma\left(g_{i_{1}}, \ldots, g_{i_{q}}\right), g_{1}, \ldots \hat{g}_{i_{1}} \ldots \hat{g}_{i_{q}} \ldots, g_{p+q-1}\right)} \\
& +(-1)^{p q+p+q} \sum_{1 \leq j_{1}<\ldots<j_{p} \leq p+q-1}(-1)^{j_{1}+\ldots+j_{p}-\frac{p(p+1)}{2}} \gamma\left(\beta\left(g_{j_{1}}, \ldots, g_{j_{p}}\right), g_{1}, \ldots \hat{g}_{j_{1}} \ldots \hat{g}_{j_{p}} \ldots, g_{p+q-1}\right) .
\end{aligned}
$$

In particular, when $p, q=2$ equation (3) becomes

$$
\begin{gathered}
{[\beta, \gamma]\left(g_{1}, g_{2}, g_{3}\right)=\sum_{1 \leq i_{1}<i_{2} \leq 3}(-1)^{i_{1}+i_{2}-3} \beta\left(\gamma\left(g_{i_{1}}, g_{i_{2}}\right), g_{1}, \ldots \hat{g}_{i_{1}} \ldots \hat{g}_{i_{3}} \ldots, g_{3}\right)} \\
\quad+\sum_{1 \leq j_{1}<j_{2} \leq 3}(-1)^{j_{1}+j_{2}-3} \gamma\left(\beta\left(g_{j_{1}}, g_{j_{2}}\right), g_{1}, \ldots \hat{g}_{j_{1}} \ldots \hat{g}_{j_{2}} \ldots, g_{3}\right) .
\end{gathered}
$$

Two deformations $[g, h]_{t},[g, h]_{t}^{\prime}$ are called equivalent if there exists a formal one-parameter family $\left\{\phi_{t}\right\}$ of linear transformations of $\mathfrak{g}$,

$$
\phi_{t}(g)=g+\sum_{k \geq 1} \beta_{k}(g) t^{k}
$$

such that

$$
[g, h]_{t}^{\prime}=\phi_{t}^{-1}\left[\phi_{t}(g), \phi_{t}(h)\right]_{t} .
$$

It is easy to see that equivalent deformations have cohomologous infinitesimals: that is, $\alpha_{1}-\alpha_{1}^{\prime}=\delta \beta$ in (4). Therefore, $\alpha_{1}$ (more precisely, its equivalence class $\left[\alpha_{1}\right] \in H^{2}(\mathfrak{g}, \mathfrak{g})$ ) is called the infinitesimal of the deformation. So, up to equivalence, the infinitesimal deformations of $\mathfrak{g}$ may be regarded as elements of $H^{2}(\mathfrak{g}, \mathfrak{g})$ with the obstructions to their propagation to higher-order deformations lying in $H^{3}(\mathfrak{g}, \mathfrak{g})$. If each element of $H^{2}(\mathfrak{g}, \mathfrak{g})$ is obstructed, then $\mathfrak{g}$ is called rigid, and if $H^{2}(\mathfrak{g}, \mathfrak{g})=0$ then $\mathfrak{g}$ is said to be absolutely rigid.

As it happens, all the deformations here considered are linear, so we require only the terms corresponding to $k=1$ and 2 in $(2)$ :

$$
\delta \alpha_{1}=0 \text { and } \delta \alpha_{2}=-\frac{1}{2}\left[\alpha_{1}, \alpha_{1}\right] .
$$

A jump deformation of $\mathfrak{g}$ is one such that all specializations, as $t$ varies over $\mathbb{F}$, are all isomorphic except perhaps, the specialization to $t=0$, which must be isomorphic to $\mathfrak{g}$ itself.

\section{Classification}

The following table contains information about the isomorphism classes of four-dimensional Frobenius Lie algebras over a field $\mathbb{F}$, where char $\mathbb{F} \neq 2$. Following the notation of [12], there is a single four-dimensional Lie algebra $\Phi^{\prime}$, and two families of four-dimensional Lie algebras $\Phi^{\prime \prime}$ and $\Phi^{\prime \prime \prime}$, parametrized by $\Delta \in \mathbb{F}$ and $0 \neq \varepsilon \in \mathbb{F}$, respectively. Note the distinguished $\Delta$ value of 0 , which affects the dimension of the second cohomology group. 


\begin{tabular}{|c|l|c|c|c|c|}
\hline $\mathfrak{g}$ & \multicolumn{1}{|c|}{ Commutator Relations } & $\operatorname{dim} H^{2}$ & $\operatorname{dim} H^{3}$ & Spectrum & Deformation \\
\hline$\Phi^{\prime}$ & $\begin{array}{l}{\left[e_{1}, e_{4}\right]=\left[e_{2}, e_{3}\right]=-e_{1},} \\
{\left[e_{2}, e_{4}\right]=-\frac{1}{2} e_{2},} \\
{\left[e_{3}, e_{4}\right]=-\frac{1}{2} e_{3}}\end{array}$ & 3 & 0 & $\left\{0, \frac{1}{2}, \frac{1}{2}, 1\right\}$ & Yes \\
\hline \multirow{2}{*}{$\Phi^{\prime \prime}(0)$} & $\begin{array}{l}{\left[e_{1}, e_{4}\right]=\left[e_{2}, e_{3}\right]=-e_{1},} \\
{\left[e_{2}, e_{4}\right]=-e_{3},} \\
{\left[e_{3}, e_{4}\right]=-e_{3}}\end{array}$ & 2 & 1 & $\{0,0,1,1\}$ & Yes \\
\hline \multirow{2}{*}{$\Phi^{\prime \prime}(\Delta)$} & $\begin{array}{l}{\left[e_{1}, e_{4}\right]=\left[e_{2}, e_{3}\right]=-e_{1},} \\
{\left[e_{2}, e_{4}\right]=-e_{3},} \\
{\left[e_{3}, e_{4}\right]=-e_{3}+\Delta e_{2}}\end{array}$ & 1 & 0 & $\left\{0,1, \frac{1 \pm \sqrt{1-4 \Delta}}{2}\right\}$ & Yes \\
\hline$\Phi^{\prime \prime \prime}(\varepsilon)$ & $\begin{array}{l}{\left[e_{1}, e_{3}\right]=\left[e_{2}, e_{4}\right]=-e_{1},} \\
{\left[e_{1}, e_{4}\right]=\varepsilon e_{2},} \\
{\left[e_{2}, e_{3}\right]=-e_{2}}\end{array}$ & 0 & 0 & $\{0,0,1,1\}$ & No \\
\hline
\end{tabular}

Table 1: Four-dimensional Frobenius Lie algebras

Remark 1. Note that $\Phi^{\prime \prime \prime}\left(\varepsilon_{1}\right)=\Phi^{\prime \prime \prime}\left(\varepsilon_{2}\right)$ if and only if the quotient $\varepsilon_{1} / \varepsilon_{2}$ is the square of an element in $\mathbb{F}$, and all other pairs of Lie algebras in the table are non-isomorphic. Only $\Phi^{\prime}$ and $\Phi^{\prime \prime}(\Delta)$ have deformations. $\Phi^{\prime \prime \prime}(\varepsilon)$ is organized as a parametrized family of Lie algebras, however, it cannot be represented as a formal deformation. For $\Phi^{\prime}$, there are three deformations: $\Phi_{1, t}^{\prime}, \Phi_{2, t}^{\prime}$, and $\Phi_{3, t}^{\prime}$, with deformation parameter $t$. The latter two are jump deformations - where the deformed algebras happens to be isomorphic to the initial algebra $\Phi^{\prime}$. The first deformation will become our prime example. For $\Phi^{\prime \prime}(\Delta)$, there are two deformations: $\Phi_{1, t}^{\prime \prime}(\Delta)$ and $\Phi_{2, t}^{\prime \prime}(0)$. The former exists for all values of $\Delta \in \mathbb{F}$, while the latter is a jump deformation and exists only when $\Delta=0$.

\section{Deformations}

Notation: To ease notation, we let $\left\{e_{1}, e_{2}, e_{3}, e_{4}\right\}$ be a basis for the Lie algebra $\mathfrak{g}$ under consideration and use $\Gamma^{i}$ to represent an $i$-cocycle of $\mathfrak{g}$ with coefficients in the adjoint representation of $\mathfrak{g}$.

\subsection{Cohomology}

In this section, we compute $H^{2}(\mathfrak{g}, \mathfrak{g})$ and $H^{3}(\mathfrak{g}, \mathfrak{g})$ for the Lie algebras $\mathfrak{g}$ listed in Table 1 . We provide detailed calculations for $\Phi^{\prime}$ (see Theorems 1 and 2). The calculations for the other algebras in Table 1 are similar.

Theorem 1. A basis for $H^{2}\left(\Phi^{\prime}, \Phi^{\prime}\right)$ is given by $\left\{\left[\Gamma_{1,1}^{2}\right],\left[\Gamma_{1,2}^{2}\right],\left[\Gamma_{1,3}^{2}\right]\right\}$, where the $\Gamma$ s are defined by

- $\Gamma_{1,1}^{2}$ is defined by $\Gamma_{1,1}^{2}\left(e_{2}, e_{4}\right)=e_{2}, \Gamma_{1,1}^{2}\left(e_{3}, e_{4}\right)=-e_{3}$.

- $\Gamma_{1,2}^{2}$ is defined by $\Gamma_{1,2}^{2}\left(e_{3}, e_{4}\right)=e_{2}$.

- $\Gamma_{1,3}^{2}$ is defined by $\Gamma_{1,3}^{2}\left(e_{2}, e_{4}\right)=e_{3}$.

Proof. The fact that $\Gamma^{2}$ is a cocycle gives the following conditions on its coefficients: 
- $c_{1}^{1,2}=c_{3}^{2,3}-3 c_{4}^{2,4}$

- $c_{2}^{1,2}=\frac{1}{2} c_{4}^{2,3}$

- $c_{3}^{1,2}=0$

- $c_{4}^{1,2}=0$
- $c_{1}^{1,3}=-c_{2}^{2,3}-3 c_{4}^{3,4}$

- $c_{2}^{1,3}=0$

- $c_{3}^{1,3}=\frac{1}{2} c_{4}^{2,3}$

- $c_{4}^{1,3}=0$
- $c_{1}^{1,4}=c_{2}^{2,4}+c_{3}^{3,4}$

- $c_{2}^{1,4}=\frac{1}{2} c_{2}^{2,3}+\frac{1}{2} c_{4}^{3,4}$

- $c_{3}^{1,4}=\frac{1}{2} c_{3}^{2,3}-\frac{1}{2} c_{4}^{2,4}$

- $c_{4}^{1,4}=c_{4}^{2,3}$.

If $\Gamma^{2}\left(e_{i}, e_{j}\right)=\sum_{k=1}^{4} c_{k}^{i, j} e_{k}, 1<i<j<4$ is to be a coboundary, there must exist $F^{1} \in C^{1}(\mathfrak{g}, \mathfrak{g})$ defined by $F^{1}\left(e_{\ell}\right)=\sum_{k=1}^{4} c_{k}^{\ell} e_{k}, 1<\ell<4$ such that the following conditions are satisfied:
1. $c_{1}^{1}=c_{2}^{2}+c_{3}^{3}+c_{1}^{2,3}$
5. $c_{3}^{1}=-\frac{1}{2} c_{4}^{2}+c_{3}^{2,3}$
2. $c_{3}^{4}=-\frac{1}{2} c_{1}^{2}-c_{1}^{2,4}$
6. $c_{4}^{1}=c_{4}^{2,3}$
3. $c_{1}^{3}=2 c_{2}^{4}-2 c_{1}^{3,4}$
7. $c_{4}^{2}=2 c_{4}^{2,4}$
4. $c_{2}^{1}=\frac{1}{2} c_{4}^{3}+c_{2}^{2,3}$
8. $c_{4}^{3}=2 c_{4}^{3,4}$
9. $c_{4}^{4}=-2 c_{2}^{2,4}=-2 c_{3}^{3,4}$
10. $c_{2}^{3,4}=0$
11. $c_{3}^{2,4}=0$.

Note that for a fixed $\Gamma^{2}$, we need only be careful of the choices for coefficients of $F^{1}$ as dictated by conditions 1-8. However, conditions 9-11 put independent conditions on $\Gamma^{2}$, rather than conditions on $F^{1}$. This means that the $\Gamma^{2}$ 's which do not satisfy $9-11$ have no such $F^{1}$. This gives a upper bound of three on the dimension of $H^{2}\left(\Phi^{\prime}, \Phi^{\prime}\right)$. Choosing $\Gamma_{1,1}^{2}, \Gamma_{1,2}^{2}$, and $\Gamma_{1,3}^{2}$ as above yields the theorem - it is straightforward to verify that these are non-cohomologous cocycles.

For $\Phi^{\prime}$, the following theorem asserts that the the third cohomolgoy group is trivial, so the infinitesimals given in Theorem 1 are unobstructed.

Theorem 2. $H^{3}\left(\Phi^{\prime}, \Phi^{\prime}\right)=0$.

Proof. Let $\Gamma^{3}\left(e_{i}, e_{j}, e_{\ell}\right)=\sum_{k=1}^{4} c_{k}^{i, j, \ell} e_{k}, 1<i<j<\ell<4$. By a straightforward computation, we see that, in order for $\Gamma^{3}$ to indeed be a cocycle, the following conditions on its coefficients must be satisfied:

- $c_{1}^{1,2,3}=-c_{2}^{1,2,4}-c_{3}^{1,3,4}+c_{4}^{2,3,4}$

- $3 c_{2}^{1,2,3}=-c_{4}^{1,3,4}$
- $3 c_{3}^{1,2,3}=c_{4}^{1,2,4}$

- $c_{4}^{1,2,3}=0$.

With these conditions established for $\Gamma^{3} \in Z^{3}(\Phi, \Phi)$, we will show that there exists some 2-cochain $\Gamma^{2}$ for which $\delta \Gamma^{2}=\Gamma^{3}$. If $\Gamma^{2}\left(e_{i}, e_{j}\right)=\sum_{k=1}^{4} c_{k}^{i, j}, 1<i<j<4$, and

- $c_{1}^{1,2}=2\left(c_{3}^{1,4}-c_{4}^{2,4}-c_{1}^{1,2,4}\right)$

- $c_{2}^{1,2}=\frac{1}{2} c_{4}^{1,4}-c_{2}^{1,2,4}$

- $c_{3}^{1,2}=-c_{3}^{1,2,4}$

- $c_{4}^{1,2}=-\frac{2}{3} c_{4}^{1,2,4}$
- $c_{1}^{1,3}=-2\left(c_{2}^{1,4}+c_{4}^{3,4}+c_{1}^{1,3,4}\right)$

- $c_{2}^{1,3}=-c_{2}^{1,3,4}$

- $c_{3}^{1,3}=\frac{1}{2} c_{4}^{1,4}-c_{3}^{1,3,4}$

- $c_{4}^{1,3}=-\frac{2}{3} c_{4}^{1,3,4}$
- $c_{1}^{1,4}=c_{2}^{2,4}+c_{3}^{3,4}+c_{1}^{2,3,4}$

- $c_{2}^{2,3}=2 c_{2}^{1,4}-c_{4}^{3,4}-2 c_{2}^{2,3,4}$

- $c_{3}^{2,3}=2 c_{3}^{1,4}+c_{4}^{2,4}-2 c_{3}^{2,3,4}$

- $c_{4}^{2,3}=c_{4}^{1,4}-c_{4}^{2,3,4}$.

then $\delta \Gamma^{2}=\Gamma^{3}$, and the result follows.

\subsection{Deformation and Spectrum}

The main results of this section are displayed in the table below. 


\begin{tabular}{|c|c|c|c|}
\hline $\mathfrak{g}_{t}$ & $\begin{array}{c}\text { Infinitesimals } \\
\text { (See } \\
\text { Theorem 1) }\end{array}$ & Commutator Relations & Spectrum \\
\hline$\Phi_{1, t}^{\prime}$ & $\Gamma_{1,1}^{2}$ & $\begin{array}{l}{\left[e_{1}, e_{4}\right]_{t}=\left[e_{2}, e_{3}\right]_{t}=-e_{1},} \\
{\left[e_{2}, e_{4}\right]_{t}=\left(t-\frac{1}{2}\right) e_{2},} \\
{\left[e_{3}, e_{4}\right]_{t}=-\left(t+\frac{1}{2}\right) e_{3}}\end{array}$ & $\left\{0, \frac{1}{2}-t, \frac{1}{2}+t, 1\right\}$ \\
\hline$\Phi_{2, t}^{\prime}$ & $\Gamma_{1,2}^{2}$ & $\begin{array}{l}{\left[e_{1}, e_{4}\right]_{t}=\left[e_{2}, e_{3}\right]_{t}=-e_{1},} \\
{\left[e_{2}, e_{4}\right]_{t}=-\frac{1}{2} e_{2},} \\
{\left[e_{3}, e_{4}\right]_{t}=-\frac{1}{2} e_{3}+t e_{2}}\end{array}$ & $\left\{0, \frac{1}{2}, \frac{1}{2}, 1\right\}$ \\
\hline$\Phi_{3, t}^{\prime}$ & $\Gamma_{1,3}^{2}$ & $\begin{array}{l}{\left[e_{1}, e_{4}\right]_{t}=\left[e_{2}, e_{3}\right]_{t}=-e_{1},} \\
{\left[e_{2}, e_{4}\right]_{t}=-\frac{1}{2} e_{2}+t e_{3},} \\
{\left[e_{3}, e_{4}\right]_{t}=-\frac{1}{2} e_{3}}\end{array}$ & $\left\{0, \frac{1}{2}, \frac{1}{2}, 1\right\}$ \\
\hline$\Phi_{1, t}^{\prime \prime}(\Delta)$ & $\Gamma_{2,1}^{2}$ & $\begin{array}{l}{\left[e_{1}, e_{4}\right]_{t}=\left[e_{2}, e_{3}\right]_{t}=-e_{1},} \\
{\left[e_{2}, e_{4}\right]_{t}=-e_{3},} \\
{\left[e_{3}, e_{4}\right]_{t}=(t+\Delta) e_{2}-e_{3}}\end{array}$ & $\left\{0,1, \frac{1 \pm \sqrt{1-4(\Delta+t)}}{2}\right\}$ \\
\hline$\Phi_{2, t}^{\prime \prime}(0)$ & $\Gamma_{2,2}^{2}$ & $\begin{array}{l}{\left[e_{1}, e_{2}\right]_{t}=t e_{3},} \\
{\left[e_{1}, e_{4}\right]_{t}=\left[e_{2}, e_{3}\right]_{t}=-e_{1},} \\
{\left[e_{2}, e_{4}\right]_{t}=\left[e_{3}, e_{4}\right]_{t}=-e_{3},}\end{array}$ & $\{0,0,1,1\}$ \\
\hline
\end{tabular}

Table 2: Deformations of four- dimensional Frobenius Lie algebras

Remark 2. For given $\Delta \in \mathbb{F}, \Phi_{1, t}^{\prime} \cong \Phi^{\prime \prime}(\Delta)$ when $t=\frac{\sqrt{1-4 \Delta}}{2}$. This follows from comparing the spectra of the respective Lie algebras and the fact that the category of Frobenius Lie algebras is stable under deformation.

Remark 3. Fixing a value of $\Delta \in \mathbb{F}$ and replacing the basis $\left\{e_{1}, e_{2}, e_{3}, e_{4}\right\}$ by $\left\{e_{1}^{\prime}=2 t e_{1}, e_{2}^{\prime}=-(t+\right.$ $\left.\left.\frac{1}{2}\right) e_{2}+e_{3}, e_{3}^{\prime}=\left(t-\frac{1}{2}\right) e_{2}+e_{3}, e_{4}\right\}$, we see that $\Phi^{\prime \prime}(\Delta) \cong \Phi_{1, t}^{\prime}$ for $t=\frac{\sqrt{1-4 \Delta}}{2} \neq 0$. Replacing the basis $\left\{e_{1}, e_{2}, e_{3}, e_{4}\right\}$ by $\left\{e_{1}, e_{2}, e_{3}^{\prime}=e_{3}+2 t e_{2}, e_{4}\right\}$ we see that $\Phi^{\prime} \cong \Phi_{2, t}^{\prime}$. Replacing the basis $\left\{e_{1}, e_{2}, e_{3}, e_{4}\right\}$ by $\left\{e_{1}, e_{2}^{\prime}=e_{2}+2 t e_{3}, e_{3}, e_{4}\right\}$ we see that $\Phi^{\prime} \cong \Phi_{3, t}^{\prime}$. Since $F_{1}$ and $F_{2}$ are non-cohomologous, they define inequivalent jump deformations. Replacing the basis $\left\{e_{1}, e_{2}, e_{3}, e_{4}\right\}$ by $\left\{e_{1}, e_{2}^{\prime}=e_{2}-e_{4}, e_{3}^{\prime}=e_{2}, e_{4}^{\prime}=e_{3}\right\}$, we see that $\Phi^{\prime \prime \prime}(\varepsilon) \cong \Phi_{2, t}^{\prime \prime}(0)$ for $t=-\varepsilon$.

Remark 4. We will only prove the results from the table corresponding to the Lie algebra $\Phi_{1, t}^{\prime}$. The proofs of the other cases are similar.

Theorem 3. $\Gamma_{1,1}^{2}$ is the infinitesimal of a deformation of $\Phi^{\prime}$, giving rise to the deformed algebra $\Phi_{1, t}^{\prime}$ defined by the relations
- $\left[e_{1}, e_{4}\right]_{t}=\left[e_{2}, e_{3}\right]_{t}=-e_{1}$
- $\left[e_{2}, e_{4}\right]_{t}=\left(t-\frac{1}{2}\right) e_{2}$
- $\left[e_{3}, e_{4}\right]_{t}=-\left(t+\frac{1}{2}\right) e_{3}$.

Proof. Set $\alpha_{1}=\Gamma_{1,1}^{2} \in H^{2}\left(\Phi^{\prime}, \Phi^{\prime}\right)$; that is,

$$
\text { - } \alpha_{1}\left(e_{2}, e_{4}\right)=e_{2} \quad \text { and } \quad-\alpha_{1}\left(e_{3}, e_{4}\right)=-e_{3} \text {. }
$$

Recall, that if $\alpha_{1}$ is the infinitesimal of a deformation $\sum_{i \geq 0} \alpha_{i} t^{i}$, then $\delta \alpha_{2}=-\frac{1}{2}\left[\alpha_{1}, \alpha_{1}\right]$. Calculating,

- $\left[\alpha_{1}, \alpha_{1}\right]\left(e_{1}, e_{2}, e_{3}\right)=2 \alpha_{1}\left(\alpha_{1}\left(e_{1}, e_{2}\right), e_{3}\right)-2 \alpha_{1}\left(\alpha_{1}\left(e_{1}, e_{3}\right), e_{2}\right)+2 \alpha_{1}\left(\alpha_{1}\left(e_{2}, e_{3}\right), e_{1}\right)=0$ 
- $\left[\alpha_{1}, \alpha_{1}\right]\left(e_{1}, e_{2}, e_{4}\right)=2 \alpha_{1}\left(\alpha_{1}\left(e_{1}, e_{2}\right), e_{4}\right)-2 \alpha_{1}\left(\alpha_{1}\left(e_{1}, e_{4}\right), e_{2}\right)+2 \alpha_{1}\left(\alpha_{1}\left(e_{2}, e_{4}\right), e_{1}\right)=2 \alpha_{1}\left(e_{2}, e_{1}\right)=0$

- $\left[\alpha_{1}, \alpha_{1}\right]\left(e_{1}, e_{3}, e_{4}\right)=2 \alpha_{1}\left(\alpha_{1}\left(e_{1}, e_{3}\right), e_{4}\right)-2 \alpha_{1}\left(\alpha_{1}\left(e_{1}, e_{4}\right), e_{3}\right)+2 \alpha_{1}\left(\alpha_{1}\left(e_{3}, e_{4}\right), e_{1}\right)=-2 \alpha_{1}\left(e_{3}, e_{1}\right)=0$

- $\left[\alpha_{1}, \alpha_{1}\right]\left(e_{2}, e_{3}, e_{4}\right)=2 \alpha_{1}\left(\alpha_{1}\left(e_{2}, e_{3}\right), e_{4}\right)-2 \alpha_{1}\left(\alpha_{1}\left(e_{2}, e_{4}\right), e_{3}\right)+2 \alpha_{1}\left(\alpha_{1}\left(e_{3}, e_{4}\right), e_{2}\right)=-2 \alpha_{1}\left(e_{2}, e_{3}\right)-2 \alpha_{1}\left(e_{3}, e_{2}\right)$ $=0$.

Thus, $\delta \alpha_{2}=0$ which implies that we get a deformation of $\Phi_{1, t}^{\prime}$ defined as follows:
- $\left[e_{1}, e_{4}\right]_{t}=\left[e_{2}, e_{3}\right]_{t}=-e_{1}$
- $\left[e_{2}, e_{4}\right]_{t}=\left(t-\frac{1}{2} e_{2}\right)$
- $\left[e_{3}, e_{4}\right]_{t}=-\left(t+\frac{1}{2}\right) e_{3}$.

Theorem 4. The spectrum of $\Phi_{1, t}^{\prime}$ is given by $\left\{0, \frac{1}{2}-t, \frac{1}{2}+t, 1\right\}$.

Proof. To determine the spectrum of $\Phi_{1, t}^{\prime}$, we must first determine a choice of Frobenius functional. Let $F=f_{1} e_{1}^{*}+f_{2} e_{2}^{*}+f_{3} e_{3}^{*}+f_{4} e_{4}^{*}$ and $B=b_{1} e_{1}+b_{2} e_{2}+b_{3} e_{3}+b_{4} e_{4} \in \Phi_{1, t}^{\prime} \cap \operatorname{ker}(F)$. For $F$ to be Frobenius, it must satisfy the system following system of equations:
- $F\left(\left[e_{1}, B\right]\right)=-b_{4} f_{1}=0$
- $F\left(\left[e_{3}, B\right]\right)=b_{2} f_{1}-\frac{1}{2} b_{4} f_{3}=0$
- $F\left(\left[e_{2}, B\right]\right)=-b_{3} f_{1}-\frac{1}{2} b_{4} f_{2}=0$
- $F\left(\left[e_{4}, B\right]\right)=b_{1} f_{1}+\frac{1}{2} b_{2} f_{2}+\frac{1}{2} b_{3} f_{3}=0$

must imply that $B=0$; this is accomplished by taking $f_{1}=1$ and $f_{2}=f_{3}=f_{4}=0$. Thus, $F=e_{1}^{*}$ is a Frobenius functional on $\Phi_{1, t}^{\prime}$.

Next, we need to determine the principal element $\widehat{F} \in \Phi_{1, t}^{\prime}$ corresponding to $F$. If $\widehat{F}=p_{1} e_{1}+p_{2} e_{2}+$ $p_{3} e_{3}+p_{4} e_{4}$, then it must be the case that
- $p_{4}=F\left(\left[\widehat{F}, e_{1}\right]\right)=F\left(e_{1}\right)=1$
- $p_{2}=F\left(\left[\widehat{F}, e_{3}\right]\right)=F\left(e_{3}\right)=0$
- $p_{3}=F\left(\left[\widehat{F}, e_{2}\right]\right)=F\left(e_{2}\right)=0$
- $-p_{1}=F\left(\left[\widehat{F}, e_{4}\right]\right)=F\left(e_{4}\right)=0$.

We conclude that $\widehat{F}=e_{4}$.

Finally, to determine the spectrum of $\Phi_{1, t}^{\prime}$, we calculate the spectrum of $[\widehat{F},-]: \Phi_{1, t}^{\prime} \rightarrow \Phi_{1, t}^{\prime}$. It is straightforward to show that

$$
\operatorname{ad} \widehat{F}=\left[\begin{array}{cccc}
1 & 0 & 0 & 0 \\
0 & \frac{1}{2}-t & 0 & 0 \\
0 & 0 & \frac{1}{2}+t & 0 \\
0 & 0 & 0 & 0
\end{array}\right]
$$

so that the spectrum of $\Phi_{1, t}^{\prime}$ is

$$
\left\{0, \frac{1}{2}-t, \frac{1}{2}+t, 1\right\}
$$

\section{Epilogue}

Topical investigations regarding the spectrum of a Frobenius Lie algebra have concentrated on seaweed Lie algebras (see [13]), or simply "seaweeds" (elsewhere called biparabolic [23]), and the recently introduced Lie poset algebras (see [5]). In a series of papers by Coll et al (see [7],[4], and [3]), it has been established that the unbroken spectrum property holds for all the classical and exceptional Frobenius seaweeds. 
Indeed, the interesting spectral properties of seaweeds was the impetus for the motivating question of this article. However, seaweeds appear to be cohomologically inert so cannot be deformed. ${ }^{1}$

In contrast, Lie poset algebras, which are necessarily solvable, have a rich deformation theory. However, we have no examples of deformable Frobenius Lie poset algebras. It is also worth noting that the unbroken spectrum property seems to be a property of Frobenius Lie poset algebras, although the spectrum is "binary", consisting of only 0 's and 1's (see [8],[9],[10], and [11]).

It is interesting to note that the spectrum of $\Phi^{\prime \prime}(\Delta)$ is an unbroken sequence of integers if and only if $\Delta=0$ or -2 . The proof is straightforward as follows. Recall that the spectrum of $\Phi^{\prime \prime}(\Delta)$ is $\left\{0,1, \frac{1 \pm \sqrt{1-4 \Delta}}{2}\right\}$. Thus, the spectrum of $\Phi^{\prime \prime}(\Delta)$ consists of integers if and only if $1-4 \Delta=a^{2}$, where $a$ is an odd integer. If $1-4 \Delta=a^{2}$, then the spectrum of $\Phi^{\prime \prime}(\Delta)$ is given by $\left\{0,1, \frac{1 \pm a}{2}\right\}$. When $a= \pm 1$, i.e., $\Delta=0$, the spectrum of $\Phi^{\prime \prime}(\Delta)$ is $\{0,0,1,1\}$; and when $a= \pm 3$, i.e., $\Delta=-2$, the spectrum of $\Phi^{\prime \prime}(\Delta)$ is $\{-1,0,1,2\}$. Since $\frac{1-a}{2}$ (resp. $\frac{1+a}{2}$ ) strictly decreases (resp. increases) as $a$ increases, the spectrum can only be unbroken for $a= \pm 1$ and \pm 3 , i.e., $\Delta=0$ and -2 .

Note that $\Phi^{\prime \prime}(\Delta)$, where $\Delta=0,-2$ is not a seaweed, since it deforms. And while $\Phi^{\prime \prime}(0)$ and $\Phi^{\prime \prime}(-2)$ are both solvable, neither is a Lie poset algebra of classical type since there is exactly one such four-dimensional Frobenius algebra. When the ground field is the complex numbers this algebra is isomorphic to $\Phi^{\prime \prime \prime}(\epsilon)$, for all $\epsilon \neq 0$.

\section{References}

[1] H. Adimi and A. Makhlouf. "Computing the index of Lie algebras." Proceedings of the Estonian Academy of Sciences, 59.4: 265, 2010.

[2] A. Belavin and V. Drindel'd. "Solutions of the classical Yang-Baxter equations for simple Lie algebas." Funct. Anal. App., 16: 159-180, 1982.

[3] A. Cameron and V. Coll. "The unbroken spectra of Frobenius biparabolics." Manuscript, 2019.

[4] A. Cameron, V. Coll, M. Hyatt, and C. Magnant. "The unbroken spectra of Frobenius seaweeds II: type-B and type-C." arXiv:1907.08775, July 20, 2019. To appear in J. Alg. Combinatorics.

[5] V. Coll and M. Gerstenhaber. "Cohomology of Lie semidirect products and poset algebras." J. Lie Theory, 26: 79-95, 2016.

[6] V. Coll, A. Hylton, and N. Mayers. "Seaweeds are rigid." Manuscript, 2020.

[7] V. Coll, M. Hyatt, and C. Magnant. "The unbroken spectrum of type-A Frobenius seaweeds." J. Alg. Combinatorics, 1-17, 2016.

[8] V. Coll and N. Mayers. "The index of Lie poset algebras." arXiv: 1908.06573, August 19, 2019. To appear in Journal of Combinatorial Theory, Series A.

[9] V. Coll and N. Mayers. "The index and spectrum of Lie poset algebras of type B, C, and D." arXiv:2001.11006, January 29, 2020, to appear in J. Alg. Combinatorics.

[10] V. Coll and N. Mayers. "Toral posets and the binary spectrum property." arXiv:1909.12918, September 27, 2019, to appear in Electronic Journal of Combinatorics.

[11] V. Coll, N. Mayers, and N. Russoniello. "The index of nilpotent Lie poset algebras." Linear Algebra and its Applications, 605: 118-129, 2020.

\footnotetext{
${ }^{1}$ In 2014 Gerstenhaber conjectured that seaweeds were cohomologically trivial. This was verified for type-A seaweeds by Elashvili and Rakviashvili (see [15], 2016). Subsequent work (yet to be published) by Coll et al (see [6]) establishes that for types B, C, and D seaweeds are cohomologically trivial. This latter work is the central result of Alan Hylton's Ph.D. dissertation at Lehigh University (in progress).
} 
[12] B. Csikos and L. Verhoczki. "Classification of Frobenius Lie algebras of dimension $\leq$ 6." Publicationes Mathematicae-Debrecen, 70: 427-451, 2007.

[13] V. Dergachev and A. Kirillov. "Index of Lie algebras of seaweed type." J. Lie Theory, 10: 331-343, 2000 .

[14] A. Diatta and B. Manga. "On properties of principal elements of Frobenius Lie algebras." J. Lie Theory, 24: 849-864, 2014.

[15] Elashvili, Alexander [Elashvili, Alexander G.] (GE-TBIL-RMI); Rakviashvili, Giorgi (GE-ISUSA) On regular cohomologies of biparabolic subalgebras of sl(n). (English, Georgian summaries) Bull. Georgian Natl. Acad. Sci. (N.S.) 10 (2016), no. 2, 1013.

[16] A. Fialowski and D. Fuchs. "Singular deformations of Lie algebras on an example." arXiv:qalg/9706027 June 20, 1997.

[17] T. Fox. "An introduction to algebraic deformation theory." Journal of Pure and Applied Algebra, 84: $17-41,1993$.

[18] M. Gerstenhaber and A. Giaquinto. "Boundary solutions of the classical Yang-Baxter equation." Letters Math. Physics, 40:337-353, 1997.

[19] M. Gerstenhaber and A. Giaquinto. "Graphs, Frobenius functionals, and the classical Yang-Baxter equation." arXiv:0808.2423v1, August 18, 2008.

[20] M. Gerstenhaber and A. Giaquinto. "The principal element of a Frobenius Lie algebra." Lett Math. Phys, 88: 333-341, 2009.

[21] Giaquinto, Anthony (1-MSS); Zhang, James J. (1-MI) Bialgebra actions, twists, and universal deformation formulas. (English summary) J. Pure Appl. Algebra 128 (1998), no. 2, 133151.

[22] M. Goze and E. Remm. "Contact and Frobeniusian forms on Lie groups." Differential Geometry and its Applications, 35: 74-94, 2014.

[23] A. Joseph. "On semi-invariants and index for biparabolic (seaweed) algebras, I." J. Algebra, 305(1):487-515, 2006.

[24] A. Nijenhuis and R. W. Richardson, Jr. "Deformations of Lie algebra structures." Journal of Mathematics and Mechanics, 17(1): 89-105, 1967.

[25] A.A. Kirillov. "Lectures on the orbit method." Vol. 64. American Mathematical Soc, 2004.

[26] A.I. Ooms. "On Frobenius Lie algebras." Communications in Algebra, 8: 13-52, 1980.

[27] A.I. Ooms, On Lie algebras having a primitive universal enveloping algebra, J. Algebra, 32 (1974), 488500 .

[28] D. Panyushev. "Inductive formulas for the index of seaweed Lie algebras." Moscow Mathematical Journal, 1(2):221-241, 2001.

[29] A. Voronov. "Quantizing Deformation Theory II." Pure and Applied Mathematics Quarterly, 16(1): $125-152,2020$. 


\section{Appendix A - dimension six}

The following table contains information about the isomorphism classes of non-decomposable 6-dimensional Frobenius Lie algebras over an algebraically closed field of characteristic 0 . 


\begin{tabular}{|c|c|c|c|c|c|}
\hline $\operatorname{dim}=6$ & Commutator Relations & $\begin{array}{l}\operatorname{dim} \\
H^{2}\end{array}$ & $\begin{array}{l}\operatorname{dim} \\
H^{3}\end{array}$ & Spectrum & Deformation(s) \\
\hline$\Phi_{6,1}$ & $\begin{array}{l}{\left[X_{1}, Y_{1}\right]=Y_{1},\left[X_{1}, Y_{3}\right]=Y_{3}} \\
{\left[X_{1}, Y_{4}\right]=2 Y_{4},\left[X_{2}, Y_{2}\right]=} \\
Y_{2},\left[X_{2}, Y_{3}\right]=Y_{3},\left[X_{2}, Y_{4}\right]= \\
Y_{4},\left[Y_{1}, Y_{2}\right]=Y_{3},\left[Y_{1}, Y_{3}\right]= \\
Y_{4}\end{array}$ & 0 & 0 & $0,0,0,1,1,1$ & No \\
\hline $\begin{array}{l}\Phi_{6,2}\{\xi, \eta\} \\
\xi \neq \eta\end{array}$ & $\begin{array}{l}{\left[X_{1}, Y_{1}\right]=Y_{1},\left[X_{1}, Y_{3}\right]=Y_{3}} \\
{\left[X_{1}, Y_{4}\right]=\xi Y_{4},\left[X_{2}, Y_{2}\right]=} \\
Y_{2},\left[X_{2}, Y_{3}\right]=Y_{3},\left[X_{2}, Y_{4}\right]= \\
\eta Y_{4},\left[Y_{1}, Y_{2}\right]=Y_{3}\end{array}$ & & & $\begin{array}{l}0,0, \frac{\eta-1}{\eta-\xi} \\
\frac{1-\xi}{\eta-\xi}, 1,1\end{array}$ & \\
\hline $\begin{array}{l}\Phi_{6,3}\{\xi: \eta\} \\
(\xi: \eta) \neq \\
(1: 1)\end{array}$ & $\begin{array}{l}{\left[X_{1}, Y_{1}\right]=Y_{1},\left[x_{1}, Y_{3}\right]=} \\
Y_{3},\left[X_{1}, Y_{4}\right]=Y_{4}+\xi Y_{3} \\
{\left[X_{2}, Y_{2}\right]=Y_{2}, \quad\left[X_{2}, Y_{3}\right]=} \\
Y_{3},\left[X_{2}, Y_{4}\right]=Y_{4}+\eta Y_{3} \\
{\left[Y_{1}, Y_{2}\right]=Y_{3}}\end{array}$ & & & $\begin{array}{l}0,0, \frac{\eta}{\eta-\xi} \\
\frac{\eta}{\xi-\eta}, 0,0\end{array}$ & \\
\hline $\begin{array}{l}\Phi_{6,4}(\xi: \eta) \\
(\xi: \eta) \neq \\
(0: 0)\end{array}$ & $\begin{array}{l}{\left[X_{1}, Y_{1}\right]=Y_{1}+\xi Y_{4}} \\
{\left[X_{1}, Y_{3}\right]=Y_{3},\left[X_{1}, Y_{4}\right]=Y_{4}} \\
{\left[X_{2}, Y_{1}\right]=\quad=\quad=\quad \eta Y_{4}} \\
{\left[X_{2}, Y_{2}\right]=Y_{2},\left[X_{2}, Y_{3}\right]=Y_{3}} \\
{\left[Y_{1}, Y_{2}\right]=Y_{3}}\end{array}$ & & & $0,0,0,1,1,1$ & \\
\hline $\begin{array}{l}\Phi_{6,5}(\xi: \eta) \\
\eta \neq 0\end{array}$ & $\begin{array}{l}{\left[X_{1}, Y_{1}\right]=\frac{1}{2} Y_{1}+\xi Y_{2}} \\
{\left[X_{1}, Y_{2}\right]=\frac{1}{2} Y_{2},\left[X_{1}, Y_{3}\right]=} \\
Y_{3}, \quad\left[X_{2}, Y_{1}\right]=\quad=\quad \eta Y_{2} \\
{\left[X_{2}, Y_{4}\right]=Y_{4},\left[Y_{1}, Y_{2}\right]=Y_{3}}\end{array}$ & & & $0,0, \frac{1}{2}, \frac{1}{2}, 1,1$ & \\
\hline$\Phi_{6,6}$ & $\begin{array}{l}{\left[X, Y_{1}\right]=Y_{1},\left[X, Y_{2}\right]=2 Y_{2}} \\
{\left[X, Y_{3}\right]=3 Y_{3},\left[X, Y_{4}\right]=4 Y_{4}} \\
{\left[X, Y_{5}\right]=5 Y_{5},\left[Y_{1}, Y_{2}\right]=Y_{3}} \\
{\left[Y_{1}, Y_{3}\right]=Y_{4},\left[Y_{1}, Y_{4}\right]=Y_{5}} \\
{\left[Y_{2}, Y_{3}\right]=Y_{5}}\end{array}$ & 0 & 0 & $0, \frac{1}{5}, \frac{2}{5}, \frac{3}{5}, \frac{4}{5}, 1$ & No \\
\hline$\Phi_{6,7}(\xi)$ & $\begin{array}{l}{\left[X, Y_{1}\right]=\xi Y_{1},\left[X, Y_{2}\right]=} \\
2 \xi Y_{2},\left[X, Y_{3}\right]=(1-2 \xi) Y_{3} \\
{\left[X, Y_{4}\right]=(1-\xi) Y_{4},\left[X, Y_{5}\right]=} \\
Y_{5},\left[Y_{1}, Y_{3}\right]=Y_{4},\left[Y_{1}, Y_{4}\right]= \\
{\left[Y_{2}, Y_{3}\right]=Y_{5}}\end{array}$ & & & $\begin{array}{l}0, \xi, 2 \xi \\
1-2 \xi, 1-\xi, 1\end{array}$ & \\
\hline$\Phi_{6,8}$ & $\begin{array}{l}{\left[X, Y_{1}\right]=Y_{2},\left[X, Y_{3}\right]=Y_{3}-} \\
Y_{4},\left[X, Y_{4}\right]=Y_{4},\left[X, Y_{5}\right]= \\
Y_{5},\left[Y_{1}, Y_{3}\right]=Y_{4},\left[Y_{1}, Y_{4}\right]= \\
{\left[Y_{2}, Y_{3}\right]=Y_{5}}\end{array}$ & 3 & 2 & $0,0,01,1,1$ & Yes (3) \\
\hline
\end{tabular}




\begin{tabular}{|c|c|c|c|c|c|}
\hline$\Phi_{6,9}$ & $\begin{array}{l}{\left[X, Y_{1}\right]=\frac{1}{3} Y_{1}+Y_{3},\left[X, Y_{2}\right]=} \\
\frac{2}{3} Y_{2}+Y_{4},\left[X, Y_{3}\right]=\frac{1}{3} Y_{3} \\
{\left[X, Y_{4}\right]=\frac{2}{3} Y_{4},\left[X, Y_{5}\right]=} \\
Y_{5},\left[Y_{1}, Y_{3}\right]=Y_{4},\left[Y_{1}, Y_{4}\right]= \\
{\left[Y_{2}, Y_{3}\right]=Y_{5}}\end{array}$ & 1 & 0 & $0, \frac{1}{3}, \frac{1}{3}, \frac{2}{3}, \frac{2}{3}, 1$ & Yes (1) \\
\hline$\Phi_{6,10}$ & $\begin{array}{l}{\left[X, Y_{1}\right]=\frac{1}{4} Y_{1},\left[X, Y_{2}\right]=} \\
\frac{1}{2} Y_{2},\left[X, Y_{3}\right]=\frac{1}{2} Y_{3}+Y_{2} \\
{\left[X, Y_{4}\right]=\frac{3}{4} Y_{4},\left[X, Y_{5}\right]=} \\
Y_{5},\left[Y_{1}, Y_{3}\right]=Y_{4},\left[Y_{1}, Y_{4}\right]= \\
{\left[Y_{2}, Y_{3}\right]=Y_{5}}\end{array}$ & 1 & 0 & $0, \frac{1}{4}, \frac{1}{2}, \frac{1}{2}, \frac{3}{4}, 1$ & Yes (1) \\
\hline$\Phi_{6,11}\{\xi, \eta\}$ & $\begin{array}{l}{\left[X, Y_{1}\right]=\xi Y_{1}\left[X, Y_{2}\right]=\eta Y_{2}} \\
{\left[X, Y_{3}\right]=(1-\eta) Y_{3},\left[X, Y_{4}\right]=} \\
(1-\xi) Y_{4},\left[X, Y_{5}\right]=\left[Y_{1}, Y_{4}\right]= \\
{\left[Y_{2}, Y_{3}\right]=Y_{5}}\end{array}$ & & & $\begin{array}{l}0, \xi, \eta, 1-\xi \\
1-\eta, 1\end{array}$ & \\
\hline $\begin{array}{l}\Phi_{6,12}(\xi) \cong \\
\Phi_{6,12}(1-\xi)\end{array}$ & $\begin{array}{l}{\left[X, Y_{1}\right]=\xi Y_{1},\left[X, Y_{2}\right]=} \\
\frac{1}{2} Y_{2}+Y_{3},\left[X, Y_{3}\right]=\frac{1}{2} Y_{3} \\
{\left[X, Y_{4}\right]=(1-\xi) Y_{4},\left[X, Y_{5}\right]=} \\
{\left[Y_{1}, Y_{4}\right]=\left[Y_{2}, Y_{3}\right]=Y_{5}}\end{array}$ & & & $\begin{array}{l}0, \frac{1}{2}, \frac{1}{2}, \xi \\
1-\xi, 1\end{array}$ & \\
\hline $\begin{array}{l}\Phi_{6,13}(\xi) \cong \\
\Phi_{6,13}(1-\xi)\end{array}$ & $\begin{array}{l}{\left[X, Y_{1}\right]=\xi Y_{1}+Y_{2},\left[X, Y_{2}\right]=} \\
\xi Y_{2},\left[X, Y_{3}\right]=(1-\xi) Y_{3}-Y_{4} \\
{\left[X, Y_{4}\right]=(1-\xi) Y_{4},\left[X, Y_{5}\right]=} \\
{\left[Y_{1}, Y_{4}\right]=\left[Y_{2}, Y_{3}\right]=Y_{5}}\end{array}$ & & & $\begin{array}{l}0, \xi, \xi \\
1-\xi, 1-\xi, 1\end{array}$ & \\
\hline$\Phi_{6,14}$ & $\begin{array}{l}{\left[X, Y_{1}\right]=\frac{1}{2} Y_{1}+Y_{2},\left[X, Y_{2}\right]=} \\
\frac{1}{2} Y_{2}+Y_{3},\left[X, Y_{3}\right]=\frac{1}{2} Y_{3}- \\
Y_{4},\left[X, Y_{4}\right]=\frac{1}{2} Y_{4},\left[X, Y_{5}\right]= \\
{\left[Y_{1}, Y_{4}\right]=\left[Y_{2}, Y_{3}\right]=Y_{5}}\end{array}$ & 2 & 0 & $0, \frac{1}{2}, \frac{1}{2}, \frac{1}{2}, \frac{1}{2}, 1$ & Yes $(2)$ \\
\hline$\Phi_{6,15}$ & $\begin{array}{l}{\left[X, Y_{1}\right]=\frac{1}{2} Y_{1}+Y_{4},\left[X, Y_{2}\right]=} \\
\frac{1}{2} Y_{2}+Y_{3},\left[X, Y_{3}\right]=\frac{1}{2} Y_{3} \\
{\left[X, Y_{4}\right]=\frac{1}{2} Y_{4},\left[X, Y_{5}\right]=} \\
{\left[Y_{1}, Y_{4}\right]=\left[Y_{2}, Y_{3}\right]=Y_{5}}\end{array}$ & 4 & 0 & $0, \frac{1}{2}, \frac{1}{2}, \frac{1}{2}, \frac{1}{2}, 1$ & Yes (4) \\
\hline
\end{tabular}

Table 3: Six-dimensional Frobenius Lie algebras

If the parameters $\xi, \eta \in \mathbb{F}$ are separated by a colon, the isomorphism class does not change if both parameters are multiplied by a non-zero number. There are curly brackets around the parameters when the isomorphism class does not depend on the order of the parameters. The algebra $\Phi_{6,11}\{\xi, \eta\}$ depends only on the set $\{\xi, 1-\xi, 1-\eta\}$. Except for these isomorphisms, the isomorphism classes of listed in the table are pairwise distinct.

Remark 5. The reader will have no difficulty establishing that all deformations associated with Table 3 are linear. 
Example 1. Consider the family given by $\Phi_{6,12}(\xi)$. Routine calculation yield:

$$
\operatorname{dim} H^{2}\left(\Phi_{6,12}(\xi), \Phi_{6,12}(\xi)\right)= \begin{cases}4, & \xi=0,1 \\ 3, & \xi=\frac{1}{4}, \frac{3}{4} \\ 6, & \xi=\frac{1}{2} \\ 2, & \text { otherwise }\end{cases}
$$

and

$$
\operatorname{dim} H^{3}\left(\Phi_{6,12}(\xi), \Phi_{6,12}(\xi)\right)= \begin{cases}2, & \xi=-1,0,1,2 \\ 1, & \xi=-\frac{1}{4}, \frac{1}{4}, \frac{3}{4}, \frac{5}{4} \\ 0, & \text { otherwise. }\end{cases}
$$

Note that the number of deformations of $\Phi_{6,12}(\xi)$ corresponds exactly to $\operatorname{dim} H^{2}$ for each value of $\xi$, and each deformation is linear. We illustrate one such deformation as follows.

Let $\Phi_{6,12, t}(2)$ be one of the two deformations given by $\Phi_{6,12}(\xi)$ when $\xi=2$. This deformation family is defined by the following collection of commutator relations:

- $\left[e_{1}, e_{2}\right]_{t}=\left(2+\frac{t}{7}\right) e_{2}$

- $\left[e_{1}, e_{3}\right]_{t}=\left(\frac{1}{2}-\frac{3 t}{7}\right) e_{3}+e_{4}$

- $\left[e_{1}, e_{4}\right]_{t}=\left(\frac{1}{2}-\frac{3 t}{7}\right) e_{4}$
- $\left[e_{1}, e_{5}\right]_{t}=(-1-t) e_{5}$

- $\left[e_{1}, e_{6}\right]_{t}=\left(1-\frac{6 t}{7}\right) e_{6}$

- $\left[e_{2}, e_{5}\right]_{t}=e_{6}$

- $\left[e_{3}, e_{4}\right]_{t}=e_{6}$ 